\title{
Sensorimotor Experience and Its Metrics: Informational Geometry and the Temporal Horizon
}

\author{
Chrystopher L. Nehaniv \\ School of Computer Science, Algorithms \& Adaptive Systems Research Groups \\ University of Hertfordshire, College Lane, Hatfield Herts AL10 9AB, United Kingdom \\ C.L.Nehaniv@herts.ac.uk
}

\begin{abstract}
We introduce metrics on sensorimotor experience at various temporal scales based on informationtheory. Sensorimotor variables through which the experience of an agent flows are modeled as information sources in the sense of Shannon information theory. Information distance between the constellation of an embodied agent's sensorimotor variables at different moments in time can be taken variable-by-variable or between entire sets of such variables to yield two classes of metrics on sensorimotor experience: the temporal experiential information distance and the Hausdorff metric on experience. Unlike mutual information, these measures each satisfy the metric axioms and thus induce a geometry on the space of experiences with the same temporal scope. Continuity of maps between experiential spaces as well as robotic applications and extensions are discussed.
\end{abstract}

\section{Motivation}

Enactive and dynamical systems views of interaction of embodied organisms, robots, and agents suggest that development and learning can be viewed in dynamical terms as the activity over time of dynamical systems structurally coupled to their environment, including the social environment $[5,18,15,3,8,2]$. This activity is understood via transitions between various kinds of interaction patterns representing characteristic "attractors" in the space of dynamical behaviours, as is the growth of the set of possible interactions and behaviours in the unfolding of a dynamical system corresponding to the agent in the course of its ontogeny. Exploration of this high-dimensional space, involving re-experiencing, predicting and varying actions in familiar behaviours is seen as the means to scaffold the agent's development and learning. How can we begin to realize this in artificial agents such as robots?

\subsection{Sensorimotor Experience}

Sensorimotor experience of embodied agents has however remained an imprecise notion. Efforts at characterizing agent-environment interactions show that it is possible to do so quantitatively using information-theoretic measures $[17,12,16,11,6]$. Consideration of sensorimotor variables modeled as random variables has also recently allowed for the import of much stronger mathematical techniques into this area the theory of metric spaces, by using the the information distance between pairs of sensorimotor information sources [12, 11, 6, 4]. Information distance between such sources, defined as their joint entropy minus their mutual information, satisfies the axioms for a metric under the identification of informationally equivalent sources [1].

Here we consider metrics on experience naturally derived from information distance, not on single information sources, but on constellations of them comprising the collection of variables describing sensorimotor an agent's connection with the world. ${ }^{1}$ Intuitively, we consider all the sensorimotor variables of an organism or agent - the sensor and effector variables the agent can "read" or "set" - as information source random variables. Locally in time for a particular agent-environment interaction, this set of random variables has certain information-theoretic characteristics that can be estimated from the joint distributions of frequencies of the particular values coming from these sensorimotor variables in parallel. Considering temporal window sizes of different sizes (temporal horizon), the frequency distributions of this collection of sensorimotor variables within the temporal horizon correspond to estimates of the grounded experiences of the agent within bounded regions of time. We define mathematical metrics between such experiences that allow us to consider temporally extended sensorimotor experiences as points in geometric space. 'Useful information' at various scales of temporal horizon (including, e.g. immediate, affective, and episodic information) for embodied organisms is systematically considered in $[9,10]$.

\subsection{Trajectories for Re-experiencing and Development}

In the metric spaces introduced here, experiences lie in an abstract geometric space. Experiences similar to a given certain experience lie within a neighborhood of it having small radius, and increasingly less similar ones lie in concentric spheres of increasing distance in this space. The difference between experiences of the same temporal scope will now be measured in bits. The agent's possible experiences are covered by the union of such small neighborhoods centered on characteristic prototypical representative experiences, and the properties of the metric could serve to guide an agent in identifying an experience as (literally) close to another it has had, or in varying some of its actions slightly to explore other experiences that are (literally) nearby in this space.

By exploring the boundary of the known dynamics in such a metric space, an artificial developing agent could potentially expand its zone of proximal development in the course of interacting with its physical and social environment, as in models of developmental psychology (cf. [19, $3])$.

\footnotetext{
${ }^{1}$ We envision future extensions that will include other internal variables less directly coupled to the environment.
} 


\section{The Geometry of Experience}

\subsection{Information Sources as Random Variables}

We consider a sensor or effector that can take on various settings or values modeled as a random variable $\mathcal{X}$ changing with time $t$, taking value $x(t) \in X$, where $X$ is the set of its possible values. For simplicity in this paper, we take time to be discrete (i.e. $t$ will denote a natural number; the set $T$ of possible times is $\mathbb{N}$ ) and allow $\mathcal{X}$ to take values in a finite set or "alphabet" $X=\left\{x_{1}, \ldots, x_{m}\right\}$ of possible values. (The approach can be generalized to continuous time and value sets with appropriate changes.)

\subsection{Entropy and Information Distance}

The entropy $H(\mathcal{X})$ of a sensor or actuator $\mathcal{X}$ is then $H(\mathcal{X})=-\sum_{x \in X} p(x) \log _{2} p(x)$, where $p(x)$ gives the probability of value $x$ being taken. Entropy is the information-theoretic measure of uncertainty introduced by Claude Shannon [14] and its units are bits.

If $\mathcal{X}$ and $\mathcal{Y}$ are jointly distributed random variables, the conditional entropy $H(\mathcal{X} \mid \mathcal{Y})$ of $\mathcal{X}$ given $\mathcal{Y}$ is the amount of uncertainty that remains about the value $\mathcal{X}$ given that the value of $\mathcal{Y}$ is known, weighted by the probability of the occurrences of particular values of $\mathcal{Y}$. This is computed to be:

$$
H(\mathcal{X} \mid \mathcal{Y})=-\sum_{x \in X} \sum_{y \in Y} p(x, y) \log _{2} p(x \mid y),
$$

where $p(x, y)$ is given by the joint distribution of $\mathcal{X}$ and $\mathcal{Y}$, and $p(x \mid y)=\frac{p(x, y)}{p(y)}$ is the conditional probability of $x$ given $y$. The information distance between $\mathcal{X}$ and $\mathcal{Y}$ is

$$
d(\mathcal{X}, \mathcal{Y})=H(\mathcal{X} \mid \mathcal{Y})+H(\mathcal{Y} \mid \mathcal{X})
$$

This satisfies the mathematical axioms for a metric:

1. $d(\mathcal{X}, \mathcal{Y})=0$ if and only if $\mathcal{X}$ and $\mathcal{Y}$ are equivalent. ${ }^{2}$

2. $d(\mathcal{X}, \mathcal{Y})=d(\mathcal{Y}, \mathcal{X})$ (symmetry)

3. $d(\mathcal{X}, \mathcal{Y}) \leq d(\mathcal{X}, \mathcal{Z})+d(\mathcal{Z}, \mathcal{Y})$ (triangle inequality)

The satisfaction of these axioms is shown by Crutchfield [1]. Thus $d$ defines a geometric structure on any space of jointly distributed information sources.

Note that there is no temporal structure present here.

\subsection{Experiential Variables and the Temporal Horizon}

For a particular agent, in a particular environment, consider one of its sensorimotor variables $\mathcal{X}$ in the context of the particular environment and beginning from a particular moment $t$ until a later moment $t+h(h>0)$. We regard the sequence of values $\mathcal{X}(t+i), 0 \leq i<h$ taken by information source $\mathcal{X}$ as deriving from a new, 'temporally and interactionally local' random variable $\mathcal{X}_{t, h}$. The $h$-tuple of values of $\mathcal{X}$ in this sequence is used to estimate the probability distribution this variable. $\mathcal{X}_{t, h}$ is the called the experiential variable with temporal horizon $h$ for sensor [resp.

\footnotetext{
${ }^{2}$ For information sources, "equivalence" refers to re-coding equivalence. That is, the values of $\mathcal{X}$ are a function of those of $\mathcal{Y}$ and vice versa. See [1].
}

actuator] $\mathcal{X}$ starting at time $t$. The sequence of $(x, y)$ pairs of values taken on by sensorimotor variables $\mathcal{X}$ and $\mathcal{Y}$ in this time window can be used as an estimate of the joint distribution of $\mathcal{X}_{t, h}$ and $\mathcal{Y}_{t, h}$. Similarly, for more variables.

Note that the degree of stationarity of the variables, the length of the temporal horizon, and the number of variables and the number of values they each can take, are all factors that may influence the accuracy of such estimates.

Due to the estimation method just described, the estimates are stationary random variables $\hat{\mathcal{X}}_{t, h}$ with every $p(x)$ a multiple of $\frac{1}{h}$, although the true $\mathcal{X}_{t, h}$ might have neither of these properties. We remark that for a finite number of sensorimotor variables taking values from finite sets over a finite temporal horizon, there are only a finite number of random variables having the above two properties that can arise by such estimation.

\subsection{Time Shifted Sensorimotor Variables}

For a particular agent, in a particular environment, consider two of its sensorimotor variables $\mathcal{X}$ and $\mathcal{Y}$. (Possibly $\mathcal{X}=$ $\mathcal{Y}$.) Consider the values taken by $\mathcal{X}$ beginning at time $t_{0}$ and those of $\mathcal{Y}$ beginning at time $t_{1}$. (Possibly $t_{0}=t_{1}$.) Consider the two-component variable $\mathcal{X}_{t_{0}, h} \times \mathcal{Y}_{t_{1}, h}$, taking values $\left(x\left(t_{0}+i\right), y\left(t_{1}+i\right)\right) \in X \times Y$. These are values whose first component comes from $\mathcal{X}$, starting from time $t_{0}$, and second component comes from $\mathcal{Y}$ with a temporal shift of $t_{1}-t_{0}$ units, i.e. starting from time $t_{1}$.

For a temporal horizon of window size $h$, we estimate the probability distribution of $\mathcal{X}_{t_{0}, h}$ in this temporal window, the time interval $\left[t_{0}, t_{0}+h\right)$, by measuring the frequency distribution of its values taken during that time. Similarly, for $\mathcal{Y}_{t_{1}, h}$ during $\left[t_{1}, t_{1}+h\right)$. And hence, we can also estimate their probability joint time-shifted distribution and the information distance $d\left(\mathcal{X}_{t_{0}, h}, \mathcal{Y}_{t_{1}, h}\right)$ using between $\mathcal{X}$ during the first time window and $\mathcal{Y}$ during the second time window by measuring the frequencies of occurrence of values $\left(x_{t_{0}+i}, y_{t_{1}+i}\right)$ as $i$ runs from 0 to $h-1 .^{3,4}$

\subsection{Experience Metric}

Consider the set of all sensorimotor variables available to an agent. Suppose there are $N$ such, $\mathcal{X}^{1}, \ldots, \mathcal{X}^{N}$. Let $E_{t, h}=\left(\mathcal{X}_{t, h}^{1}, \ldots, \mathcal{X}_{t, h}^{N}\right)$ be the (ordered) set of these variables considered over a temporal window of size $h$ starting at $t$. We call $E_{t, h}$ the agent's experience from time $t$ having temporal horizon $h$. The set of all possible experiences of temporal horizon $h$ is denoted $\mathcal{E}_{h}$.

Let $E=E_{t, h}$ and $E^{\prime}=E_{t^{\prime}, h}$ be experiences of an agent from time $t$ and $t^{\prime}$, respectively, both with horizon size $h$. We shall often suppress the subscripts $t$ or $h$ in our notation

\footnotetext{
${ }^{3}$ As above without temporal shifting, clearly there are issues related to the size of the temporal horizon $h$ and also the number of values $\mathcal{X}$ and $\mathcal{Y}$ may take that affect the accuracy of these estimates. Also in practice, independent samples of time shifted sensorimotor variables are not available.

${ }^{4}$ Previous work on sensory reconstruction and sensorimotor learning on robots by Olsson et al. [12] generally uses $t_{0}=0$ and $h$ the largest available, but also has considered shifts by a small amount $t_{1}-t_{0}$ to study temporal correlations in information such as occur, e.g., in optical or tactile flow [13].
} 
when their value is free or can be determined from context. Sometimes we write $E(t)$ or $E_{h}(t)$ for $E_{t, h}$. Define a metric on experiences of temporal horizon $h$ as

$$
D\left(E, E^{\prime}\right)=\sum_{k=1}^{N} d\left(\mathcal{X}_{t, h}^{k}, \mathcal{X}_{t^{\prime}, h}^{k}\right),
$$

where $d$ is the information distance.

We remark that the experience metric defined here is the metric induced by taking the $\mathrm{N}$-fold direct product of the space of information sources with itself and restricting to $N$-tuples whose components always arise from the sensorimotor variables in the same order, all having the same starting time and temporal horizon.

Theorem $1 D$ is a indeed metric on the set of experiences of fixed temporal horizon $h$ for a given agent.

Proof: That the metric axioms hold for $D$ follows from the fact that they hold componentwise, since $d$ is a metric.

Corollary $1 \bar{D}=\frac{1}{N} D$, the average experiential information distance per sensorimotor variable, is a metric on the set of experiences of fixed temporal horizon $h$ for a given agent.

Thus $D$ (or $\bar{D}$ ) provides a geometric structure on the set $\mathcal{E}_{h}$ of experiences of an agent. The units of $D$ are bits and those of $\bar{D}$ are bits per sensorimotor variable.

\subsection{The Local Picture at an Experience}

For any subset $S$ of the set sensorimotor variables $V$, let $E^{S}$ be derived from experience $E$ be omitting all components from random variables not in $S$. Then, we have a metric on the set $\mathcal{E}^{S}$ of experiences of the agent restricted to variables $S$ :

$$
D^{S}\left(E^{S}, E^{\prime S}\right)=\sum_{\mathcal{X}^{k} \in S} d\left(\mathcal{X}_{t, h}^{k}, \mathcal{X}_{t^{\prime}, h}^{k}\right)
$$

Clearly $D^{V}=D$. The following is obvious (by definition). Lemma 1 For any partition of the set of sensorimotor variables $V$ into two subsets $S$ and $M$ (i.e. with $S \cap M=\emptyset$, $S \cup M=V$ ), we have $D=D^{S}+D^{M}$, or, more precisely, $D\left(E_{t, h}, E_{t^{\prime}, h}\right)=D\left(E_{t, h}^{S}, E_{t^{\prime}, h}^{S}\right)+D\left(E_{t, h}^{M}, E_{t^{\prime}, h}^{M}\right)$.

Similar facts hold for partitions of $V$ into more subsets.

In what follows, we take $M$ to be the set of proprioceptive sensors (read-write random variables, giving the agent's internal state and effector settings) and $S$ to be set of exteroceptive variables (read-only variables, corresponding to sensors).

The local picture of experience at time $t_{0}$ is the trajectory plot of the distance $D^{S}$ of the sensory experience $E^{S}(t)$ to $E^{S}\left(t_{0}\right)$ vs. the distance $D^{M}$ of motor experience $E^{M}(t)$ to $E^{M}\left(t_{0}\right)$ as time $t$ varies over the experience of the agent; that is, experience at time $t$ is plotted as $\left(D^{S}\left(E_{t, h}^{S}, E_{t_{0}, h}^{S}\right), D^{M}\left(E_{t, h}^{M}, E_{t_{0}, h}^{M}\right)\right)$. By the Lemma, the distance in $\mathcal{E}$ of any experience at $t$ from the experience at time $t_{0}$ is its the sum of its $\mathrm{x}$ - and $\mathrm{y}$-displacements from the origin in the local picture.
Remarks. (1) Distances from points in the plot from experience at $t_{0}$, the origin, are faithfully represented in the local picture. (2) Distances $D\left(E_{t, h}, E_{t^{\prime}, h}\right)$ between other points with $t, t^{\prime} \neq t_{0}$ are not necessarily faithfully represented in the local picture, but are not closer than they appear, since by the triangle inequality, we have for all times $t$ and $t^{\prime}$,

$$
D\left(E(t), E\left(t^{\prime}\right)\right) \leq D\left(E(t), E\left(t_{0}\right)\right)+D\left(E\left(t_{0}\right), E\left(t^{\prime}\right)\right) .
$$

Thus the right hand side of the inequality, the sum of the distances of $t$ and $t^{\prime}$ to $t_{0}$, is an upper bound for the experiential distance between $t$ and $t^{\prime}$. (3) The set of experiences

$$
B_{r}\left(E\left(t_{0}\right)\right)=\left\{E(t): D\left(E\left(t_{0}\right), E(t)\right)<r\right\}
$$

less than $r$ bits from $E\left(t_{0}\right)$ are contained within the triangle bounded by the origin, $(r, 0)$ and $(0, r)$ in the local picture. $B_{r}\left(E\left(t_{0}\right)\right)$ is called the ball of radius $r$ centered at $E\left(t_{0}\right)$.

\subsection{Continuous and Contracting Experiential Maps}

The fact that $D$ is a metric on experience gives a welldefined notion of continuous mapping on spaces of experiences. Suppose $\mathcal{E}$ and $\mathcal{F}$ are spaces of experiences of the same or different agents, of the same or different temporal scope, and $F: \mathcal{E} \rightarrow \mathcal{F}$ is a function that associates to each experience $E \in \mathcal{E}$ an experience $F(E) \in \mathcal{F}$ such that for all $\epsilon>0$, there is a $\delta>0$, such that $D\left(E, E^{\prime}\right)<\delta$ implies $D\left(F(E), F\left(E^{\prime}\right)\right)<\epsilon$, then $F$ is called continuous. Equivalently, continuity of $F$ means that if $F$ maps $E$ to $F(E)$, then $F$ given any radius ball $B_{\epsilon}(F(E))$ centered at $F(E)$, no matter how small, must map some ball $B_{\delta}(E)$ centered at $E$ to inside of $B_{\epsilon}(F(E))$. In other words, $F\left(B_{\delta}(E)\right) \subseteq B_{\epsilon}(F(E))$.

For the metric space $\mathcal{E}_{h}$ of experiences with fixed temporal horizon $h$, the experiential trajectory of an agent is the continuous function from $T$ to $\mathcal{E}_{h}$ taking time $t$ to $E_{t, h}$ The time-horizon $h$ graph of the experience trajectory is

$$
\Gamma_{h}=\left\{\left(t, E_{t, h}\right): t \in T\right\} \subseteq T \times \mathcal{E}_{h} .
$$

\section{Examples of Continuous Maps.}

1. (Sensorimotor Variable Restriction). If $V^{\prime}$ is a subset of the set of sensorimotor variables $V$, then function mapping the experience of all variables in $V$ to the restricted experiences that only consider the variables in $V^{\prime}$ is a continuous experiential map $E \mapsto E^{V}$. More generally, if $V^{\prime} \subseteq W$ are any subsets of an agent's sensorimotor variables, then the function $E^{W} \mapsto E^{V^{\prime}}$ is continuous.

2. (Temporal Extension). When $h^{\prime} \geq h$ are temporal horizon sizes, define a function mapping the graph of experience with temporal horizon $h$ to experiences of longer temporal horizon $h^{\prime}$ by $e\left(t, E_{t, h}\right)=E_{t, h^{\prime}}$. This function $e: \Gamma_{h} \rightarrow \mathcal{E}_{h^{\prime}}$ is well-defined ${ }^{5}$ and continuous.

Proof of (1): Since $V^{\prime} \subseteq V$, we have $D^{V^{\prime}}\left(E^{V^{\prime}}, E^{\prime V^{\prime}}\right) \leq$ $D\left(E, E^{\prime}\right)$. So for each $\epsilon>0$, taking $\delta=\epsilon$, we have that $D\left(E, E^{\prime}\right)<\delta$ implies $D^{V^{\prime}}\left(E^{V^{\prime}}, E^{\prime V^{\prime}}\right)<\epsilon$. The more general case is similar. Proof of (2) is omitted here.

\footnotetext{
${ }^{5}$ Note that mapping $E_{t, h}$ to $E_{t, h^{\prime}}$ would not be a well-defined function if $E_{t, h}=E_{t^{\prime}, h}$ for some $t \neq t^{\prime}$ but $E_{t, h^{\prime}} \neq E_{t^{\prime}, h^{\prime}}$.
} 
3. (Contractions). Given $\mathcal{E}$ is a metric space of experiences, a function $F: \mathcal{E} \rightarrow \mathcal{E}$ is called a contraction if there is some $c \in[0,1)$ such that for all $E, E^{\prime} \in \mathcal{E}$,

$$
D\left(F(E), F\left(E^{\prime}\right)\right) \leq c D\left(E, E^{\prime}\right) .
$$

A contraction is always continuous as can be seen by taking $\delta=c^{-1} \epsilon$ if $c \neq 0$ (or $\delta$ arbitrary if $c=0$ ).

Theorem 2 (Contraction Mapping) Suppose $\mathcal{E}$ is a metric space of experiences and $F: \mathcal{E} \rightarrow \mathcal{E}$ is a contraction. Then

$$
\lim _{n \rightarrow \infty} D\left(F^{n}(E), F^{n+1}(E)\right)=0 .
$$

Proof: By definition of contraction, we have $D\left(F^{2}(E), F(E)\right)<c D(F(E), E)$. By induction,

$$
D\left(F^{n+1}(E), F^{n}(E)\right) \leq c^{n} D\left(F^{n}(E), F^{n-1}(E)\right)
$$

holds of all $n>0$. Since $0 \leq c<1$, the limit as $n$ goes to infinity is zero.

\subsection{Hausdorff Experience Metric}

Each of the random variables $\mathcal{X}_{t, h}^{i}$ are points in the geometric space of information sources, for every sensor/actuator (indexed by $i$ ), time $t$, and temporal horizon $h$. By regarding the (unordered) experiential set $C_{t, h}=\left\{\mathcal{X}_{t, h}^{1}, \ldots, \mathcal{X}_{t, h}^{N}\right\}$ as a subset of the geometric space of information sources whose metric is the information distance $d$, we can use the natural construction of the Hausdorff metric induced by $d$ on subsets of this metric space. Let $C=C_{t, h}$ and $C^{\prime}=C_{t^{\prime}, h}^{\prime}$ be two experiential sets of temporal horizon $h$ for a given agent. Define $\delta\left(C, C^{\prime}\right)=\max _{\mathcal{X} \in C} \min _{\mathcal{Y} \in C^{\prime}} d(\mathcal{X}, \mathcal{Y})$, where $d$ is information distance. ${ }^{7}$ Then

$$
d_{H}\left(C, C^{\prime}\right)=\max \left(\delta\left(C, C^{\prime}\right), \delta\left(C^{\prime}, C\right)\right)
$$

is the Hausdorff metric on experiential sets.

More generally, let $\mathcal{E}$ be the set of all non-empty, bounded experiential sets of given temporal horizon $h$ for a given agent (or agents, if their sensorimotor variables jointly distributed at all moments of time under consideration).

Theorem 3 The Hausdorff experiential metric $d_{H}$ is a metric on the set $\mathcal{E}$ of (unordered) bounded experiential sets with temporal scope $h$.

Proof: The proof follows a standard construction of Hausdorff. Obviously, $d_{H}$ is symmetric. If $d_{H}\left(C, C^{\prime}\right)=0$ then $\delta\left(C, C^{\prime}\right)=0$ implying for each $\mathcal{X} \in C$ that the minimum of $d(\mathcal{X}, \mathcal{Y})$ as $\mathcal{Y}$ ranges over $C^{\prime}$ is zero, i.e. each $\mathcal{X} \in C$ is re-coding equivalent to something in $C^{\prime}$, whence $C \subseteq C^{\prime}$

\footnotetext{
${ }^{6}$ This is a weakening of the standard contraction mapping theorem for complete metric spaces, which guarantees the existence of a fixed point for any contraction. The experiential metric spaces in general have not been shown to be complete.

${ }^{7}$ The definition of $d_{H}$ here has been simplified by using max and min instead of sup and inf. Given that only a finite number of estimate random variables with a fixed temporal horizon are accessible to the agent, this is justified by operational considerations where $C$ and $C^{\prime}$ are always finite.
}

Table 1: AIBO Telemetry Collected

\begin{tabular}{|l|r|l|r|}
\hline Sensors & $\#$ & Motors & $\#$ \\
\hline IR-Distance & 1 & Leg Joint Positions & 12 \\
Accelerometers & 3 & Head Joint Positions & 4 \\
Temperature/Battery & 2 & Tail Joint Positions & 2 \\
Buttons & 8 & Motor Force / Duties & 18 \\
Visual & 27 & & \\
\hline Total Sensors & 41 & Total Motors & 36 \\
\hline
\end{tabular}

(up to informational equivalence). Similarly, $C^{\prime} \subseteq C^{\prime}$, so $C$ and $C^{\prime}$ consist of equivalent information sources. The triangle inequality can be shown for $\delta$, and hence for $d_{H}$, using the properties of min and max.

Remarks. (1) Note the unlike the experience metric $D$, the Hausdorff experience metric can be used to compare sets of sensorimotor variables that do not necessarily have the same number of variables. (2) Considerations of continuity of maps to or from the space of experiences with the Hausdorff experiential metric, as well as contractions and results about them can be derived analogously as for the experience metrics of the previous section.

\section{Examples: Metrics for Robot Experience}

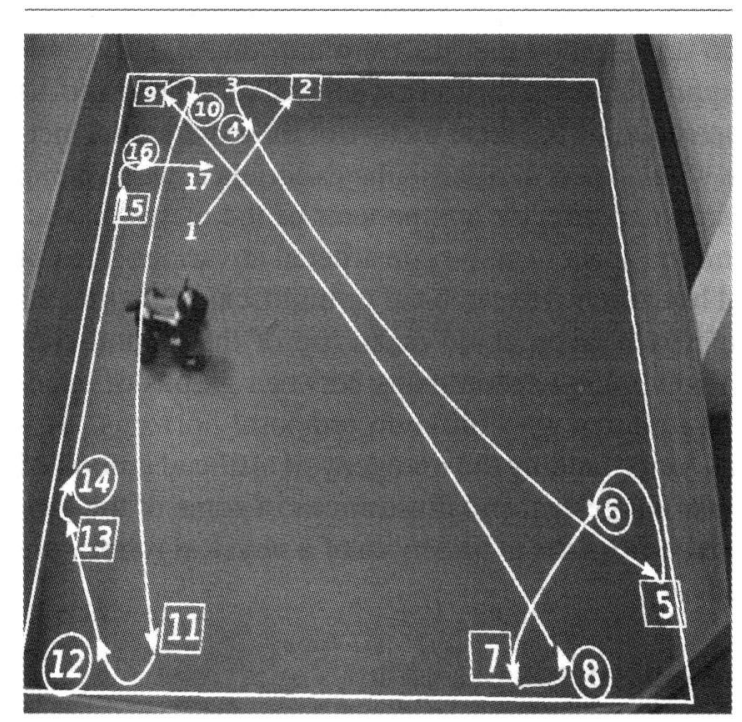

Figure 1: Trajectory of the wandering AIBO robot in $2 \mathrm{~m} \mathrm{x}$ $2 \mathrm{~m}$ arena.

\subsection{Experimental Set-up}

Sensorimotor variable data for a SONY AIBO robot wandering in a $2 \mathrm{~m} \times 2 \mathrm{~m}$ environment were gathered previously to study information distance characterizations of sensorimotor interactions [6]. These data are reanalyzed here using the tools of the experiential metric introduced above for temporal horizons of size 20,40, and 80 . More details of experiments with robots and examination of other aspects of 
the temporal experiential metric is given in the companion paper [7]. Seventy-seven sensorimotor variables were partitioned into two classes (read-only) variables (41 "sensors") and (read-write) variables (36 "motors") - see Table 1. Data from sensorimotor variables was sampled at approximately 10 times per sec. Figure 1 shows the trajectory of the robot in the arena lasting 90.3 seconds.

\subsection{Experience Metric at Different Temporal Scales}

Figures 2-6 illustrate the experiential information distance concepts discussed above for the temporal regions of experience ending at three different timesteps $t=220,250$, or 310 (rows) with increasing temporal horizon $h$ of 20,40 , and 80 time units. Time is measured in units of $100 \mathrm{msec}$.

Although there are large empty areas in the neighborhood of the sample experiences examined (with no experience within $1 \mathrm{bit} / \mathrm{variable}$ of the origin), the experiences which are nearest to $E\left(t_{0}\right)$ in the local picture are qualitatively similar to $E\left(t_{0}\right)$ from an external observer's point of view (e.g. walking, or turning if the robot was walking or turning during the temporal region at time $t_{0}$.)

\section{Summary}

We have introduced metrics for regions of experience having uniform temporal horizon grounded in the sensorimotor variables of an embodied agent. The experiential information distance, average experiential information distance, and Hausdorff experiential metrics were introduced, and continuity mapping and contraction properties were discussed, as well an introducing the local picture of the experiential metric space with respect to a particular experience, partitioning into sensory and effector components of the metric. Experiential metric spaces can be constructed at various sizes of temporal horizon $h$. Hierarchical or multiscale elaborations of the approach taken could make use of the natural continuous temporal extension maps between graphs of experiential trajectories with different temporal horizons. Applications for grounding the ontogeny of artificial embodied agents were suggested, and illustrations of some of the concepts on data from the experiences of a physical robot were presented.

\section{Acknowledgments}

The work described in this paper was conducted in part within the EU Integrated Project RовотCuв (Robotic Open-architecture Technology for Cognition, Understanding, and Behaviours) and was funded in part by the European Commission through the E5 Unit (Cognition) of FP6-IST under Contract FP6-004370. Thanks to Naeem Assif Mirza for behavioural programming and gathering of the AIBO sensorimotor data, as well as software implementation of the experience metric and generation of the plots in section 3.

\section{Bibliography}

[1] J.P. Crutchfield. Information and its metric. In L. Lam and H.C. Morris, editors, Nonlinear Structures in Physical Systems - Pattern Formation, Chaos and Waves, pages 119-130. Springer-Verlag, New York, 1990.
[2] K. Dautenhahn, B. Ogden, and T. Quick. From embodied to socially embedded agents - implications for interaction-aware robots. Cognitive Systems Research, 3(3):397-428, 2002.

[3] Kerstin Dautenhahn and Thomas Christaller. Remembering, rehearsal and empathy - towards a social and embodied cognitive psychology for artifacts. In Sean O'Nuallain and Paul Mc Kevitt, editors, Two sciences of the mind. Readings in cognitive science and consciousness, pages 257-282. John Benjamins, 1996.

[4] A. Klyubin, D. Polani, and C. L. Nehaniv. Tracking information flow through the environment: Simple cases of stigmergy. In Artificial Life IX: Proceedings of the Ninth International Conference on Artificial Life. MIT Press, 2004.

[5] Humberto R. Maturana and Francisco J. Varela. The Tree of Knowledge: The Biological Roots of Human Understanding. New Science Library (Shambhala), 1987.

[6] N. A. Mirza, C. L. Nehaniv, K. Dautenhahn, and R. te Boekhorst. Using sensory-motor phase-plots to characterise robot-environment interactions. In 6th IEEE International Symposium on Computational Intelligence in Robotics and Automation, 2005.

[7] N. A. Mirza, C. L. Nehaniv, K. Dautenhahn, and R. te Boekhorst. Using temporal information distance to locate sensorimotor experience in a metric space. In IEEE Congress on Evolutionary Computation, 2005.

[8] C. Nehaniv and K. Dautenhahn. Embodiment and memoriesalgebras of time and history for autobiographic agents. In R. Trappl, editor, Cybernetics and Systems '98: Proc. 14th European Meeting on Cybernetics and Systems Research, volume 2, pages 651-656. Vienna: Austrian Society for Cybernetic Studies, 1998.

[9] C. L. Nehaniv, K. Dautenhahn, and M. J. Loomes. Constructive biology and approaches to temporal grounding in post-reactive robotics. In G. T. McKee and P. Schenker, editors, Sensor Fusion and Decentralized Control in Robotics Systems II (September 19-20, 1999, Boston, Massachusetts), Proceedings of SPIE, volume 3839, pages 156-167, 1999.

[10] C. L. Nehaniv, D. Polani, K. Dautenhahn, R. te Boekhorst, and L. Cañamero. Meaningful information, sensor evolution, and the temporal horizon of embodied organisms. In Artificial Life VIII, pages 345-349. MIT Press, 2002.

[11] L. Olsson, C. L. Nehaniv, and D. Polani. The effects on visual information in a robot in environments with oriented contours. In Fourth International Workshop on Epigenetic Robotics (EpiRob2004), pages 83-88, 2004.

[12] L. Olsson, C. L. Nehaniv, and D. Polani. Sensory channel grouping and structure from uninterpreted sensor data. In IEEE NASA/DoD Conference on Evolvable Hardware 2004, pages 153-160. IEEE Computer Society, 2004.

[13] L. Olsson, C. L. Nehaniv, and D. Polani. Discovering motion flow by temporal-informational correlations in sensors. In Fifth International Workshop on Epigenetic Robotics (EpiRob2005), 2005.

[14] Claude E. Shannon. A mathematical theory of communication. Bell System Technical Journal, 27:379-423 and 623-656, 1948.

[15] L.B. Smith and E. Thelen. A Dynamic Systems Approach to Development. MIT Press, 1993.

[16] G. Tarapore, M. Lungarella, and G. Gómez. Fingerprinting agentenvironment interaction via information theory. In F. Groen, N. Amato, A. Bonarini, E. Yoshida, and B. Kröse, editors, Proceedings of the 8th International Conference on Intelligent Autonomous Systems, pages 512-520, 2004.

[17] I. J. A. te Boekhorst, M. Lungarella, and R. Pfeifer. Dimensionality reduction through sensori-motor coordination. In O. Kaynak, E. Alpaydin, E. Öja, and L. Xu, editors, Proceedings of the joint International Conference on Artificial Neural Networks and Neural Information Processing. Istanbul, Turkey, Lecture Notes in Computer Science 2114, pages 496-503, Berlin, 2003. Springer.

[18] Francisco J. Varela, Evan Thompson, and Eleanor Rosch. The embodied mind: Cognitive science and human experience. MIT Press, Cambridge, MA, USA., 1991.

[19] L.S. Vygotsky. Mind and society: The development of higher mental processes. Harvard University Press., Cambridge, MA, 1978. 

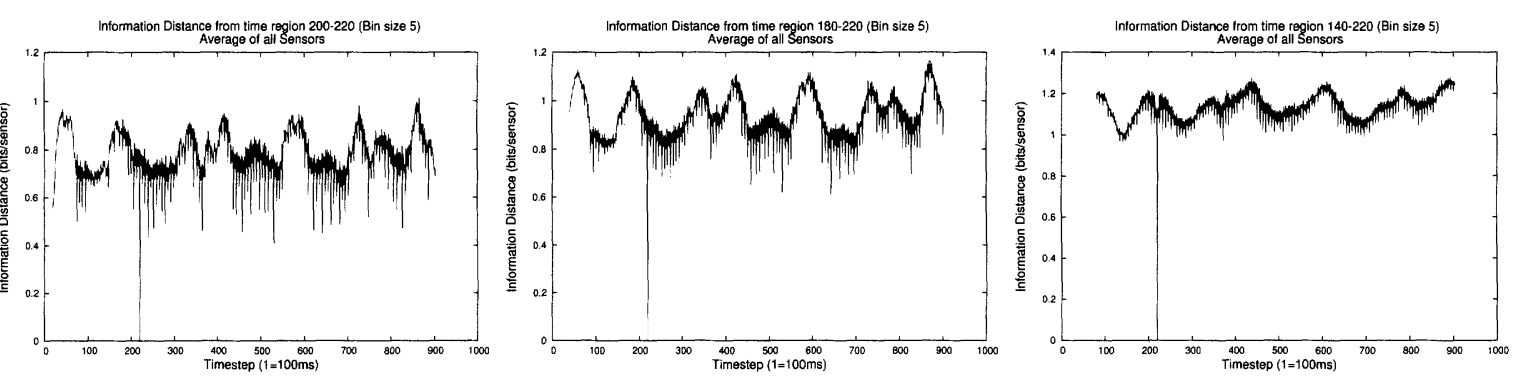

$\mathrm{t}=250$
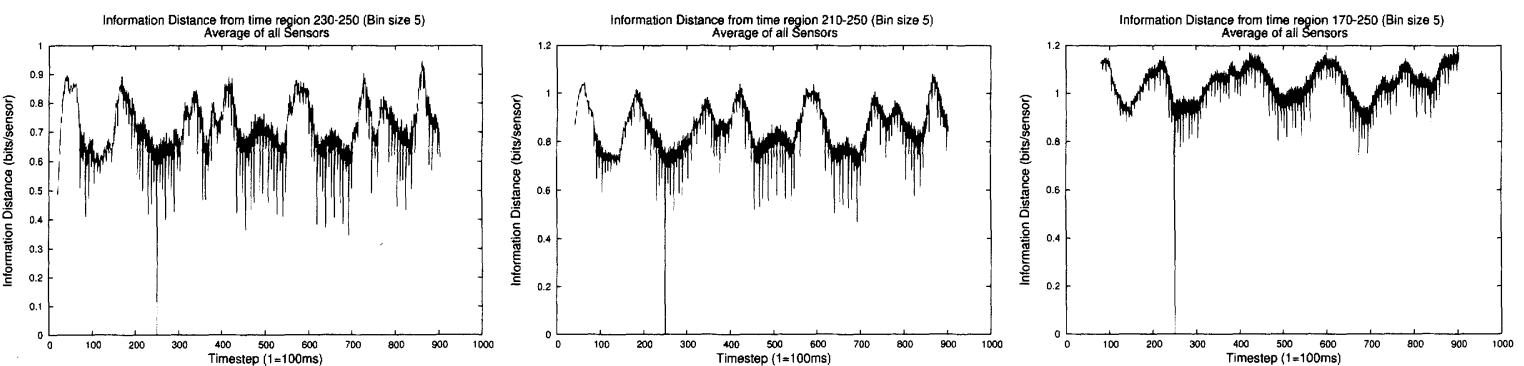

$t=310$
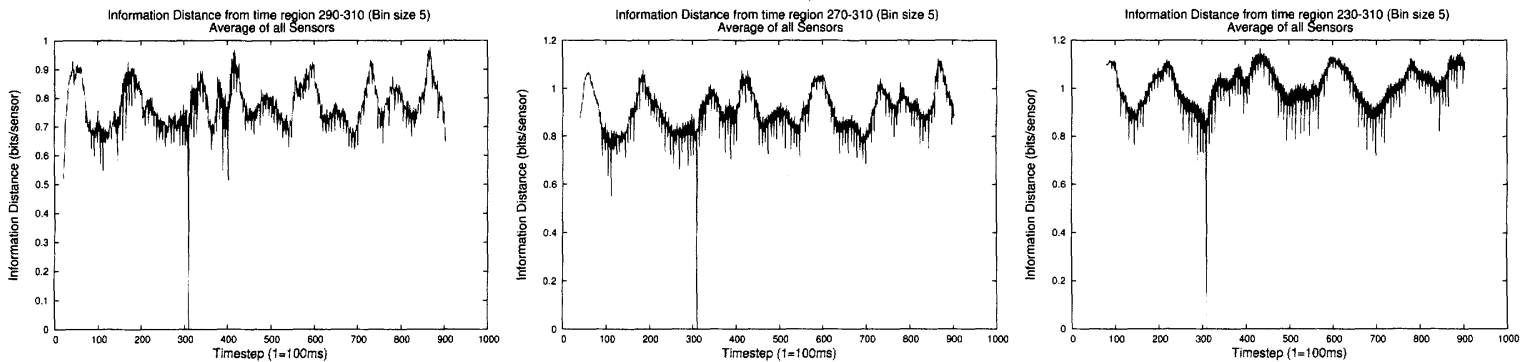

Figure 2: Distances of all sensorimotor experiences from the temporal regions of sizes 20, 40, and 80 (columns), ending at timesteps 220,250 , and 310 (rows) using $\bar{D}$ metric. Horizontal axis gives ending point of temporal region $t=220,250,310$ (rows) with increasing temporal horizon $h$. Time is measured in units of $100 \mathrm{msec}$. Vertical axis shows distance from experience $E_{t-h, h}$ in bits per sensorimotor variable. During the temporal windows shown the behaviour of the robot, from a human observer perspective, was as follows: (row 1) 200 - 220 walking, 180 - 220 walking, 140 - 220 part turning, part walking; (row 2) 230 - 250 walking, 210 - 250 walking, 170 - 250 (mostly) walking; (row 3) 290 - 310 turning, 270 - 310 walking and turning, 230 - 310 walking and turning (walking dominates). Different columns are related by continuous temporal extension maps between graphs of trajectories $i d \times e: \Gamma_{h} \rightarrow \Gamma_{h^{\prime}}$ in experiential metric spaces of increasing temporal horizon - see section 2.7. Continuity is evidenced in the the gradual change as $h$ increases. Continuity of the experiential metric $D$ is seen in the gradual changes as $t$ increases - except that no experiences appear to be extremely close to the examined experiences. This might be due to low number of samples within a window in estimating the $E_{t, h}$, or due to sparseness of the experiences sampled in this short experiment. 
Total

$\mathrm{h}=20$

$\mathrm{h}=40$
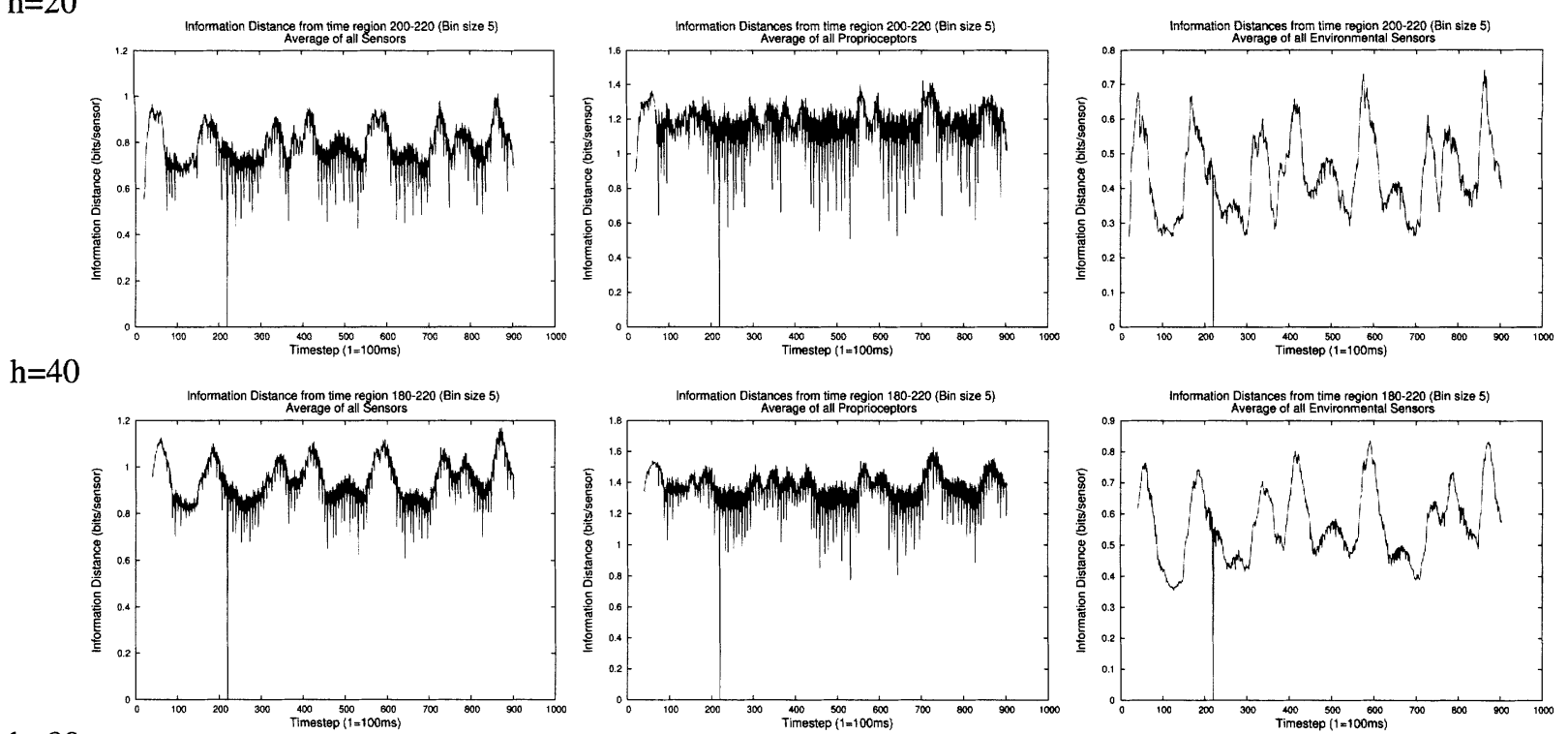

$h=80$
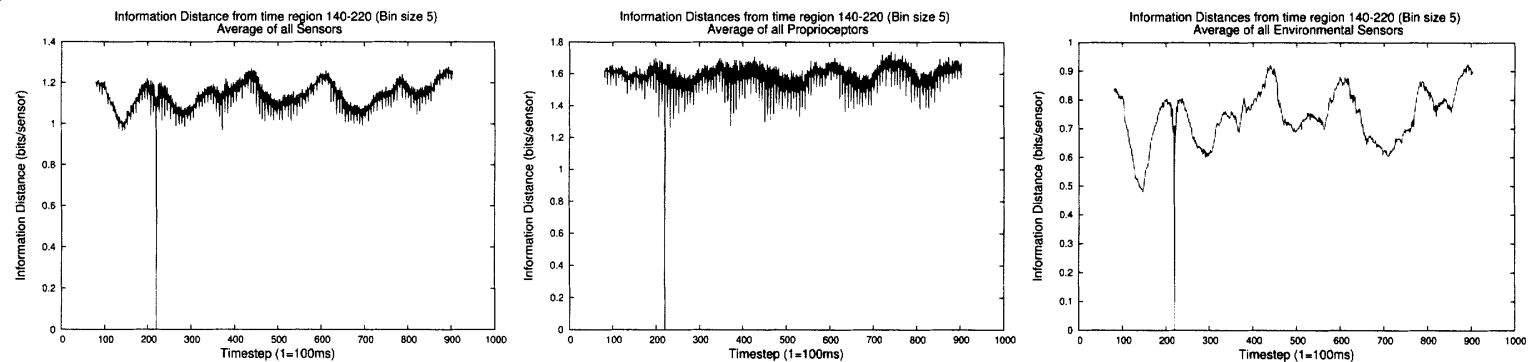

Figure 3: Distance $\bar{D}$ of sensorimotor, sensory and motor experience (columns) from the temporal regions ending at timestep 220. Horizontal axis gives ending point $t$ of temporal region. Behaviour and units as is Figure 2. Distances shown are in the experiential spaces $\mathcal{E}, \mathcal{E}^{S}$, and $\mathcal{E}^{M}$ (left to right), with with temporal horizons $h=20,40$, and 80 timesteps (top to bottom). Time units are $100 \mathrm{msec}$. The first column distance is the average weighted by number of variables of the second and third, illustrating Lemma 1.

$$
\mathrm{t}=220
$$
$\mathrm{t}=250$
$t=310$

Motor Variables
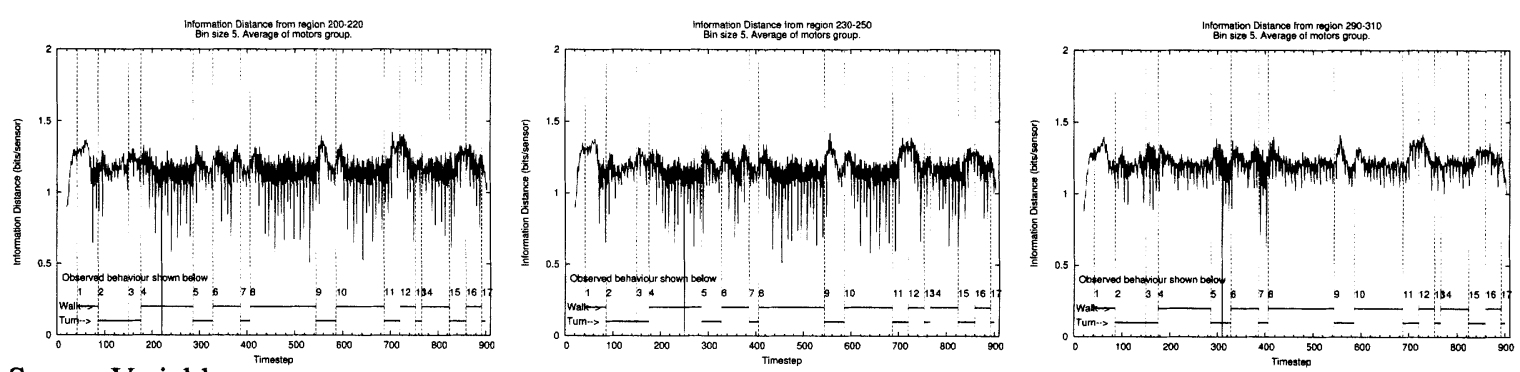

Sensor Variables
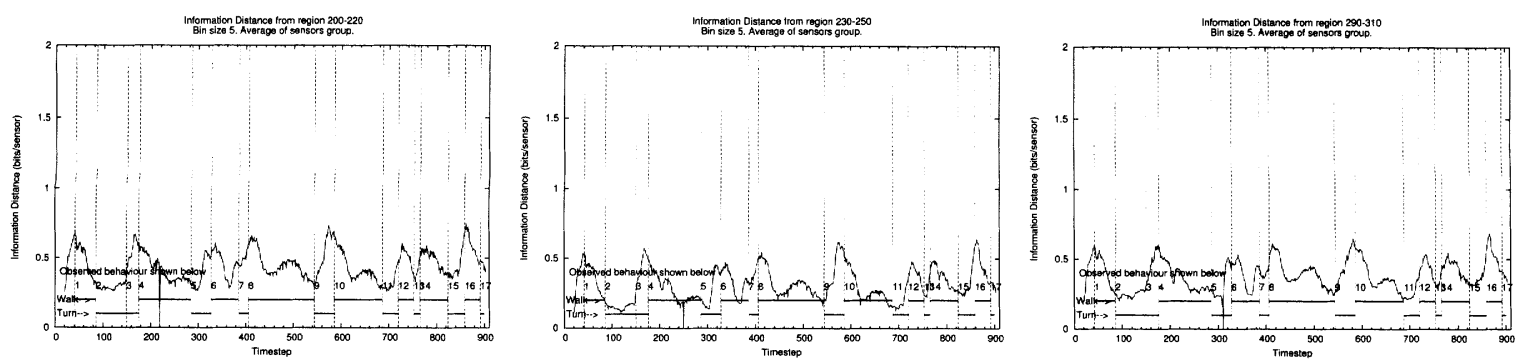

Figure 4: Motor and sensor temporal distances (rows) from time regions 220, 250 and 310 (columns) for temporal window size 20. Vertical lines indicate waypoints marked in Figure 1. Walking and turning behaviours are indicated in each graph. 

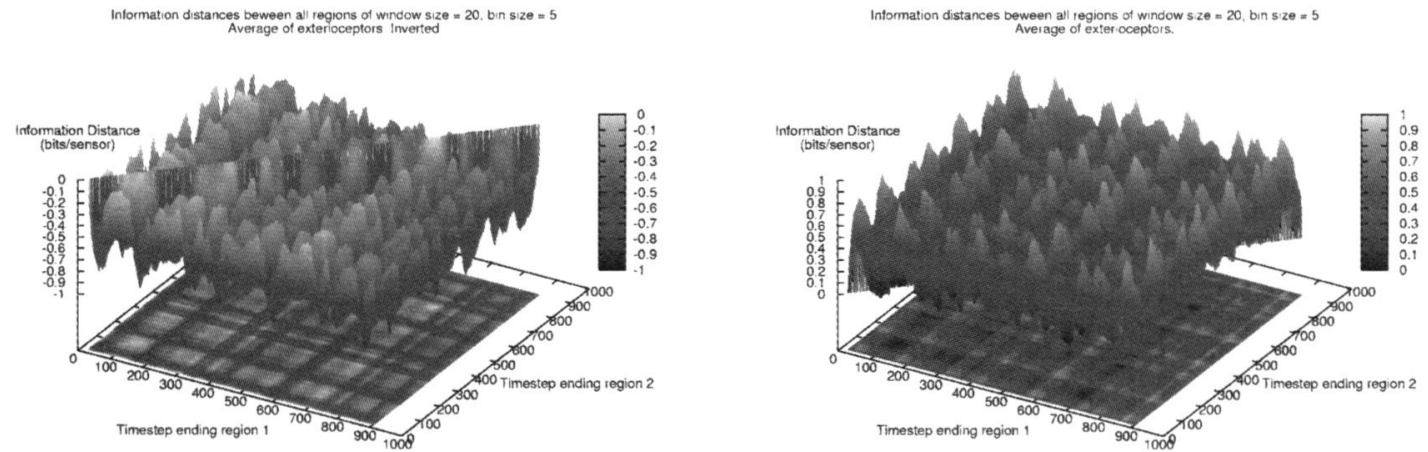

Figure 5: Sensory experiential distances between all pairs of temporal regions of window size $h=20$ in the experiential metric space $\mathcal{E}_{20}^{S}$ with metric $\bar{D}$ in bits per sensorimotor variable (vertical axis). Time units are in 100 msec. Left: nearest experiences shown uppermost (by negating the $\bar{D}$ ). Right: farthest experiences uppermost. Horizontal axes labeled by ending time of temporal regions of size 20 . The left graph shows that experiences in this experiment are only very close to themselves. Both graphs reveal an apparent nearly periodic regularity in distances to other experiences over time perhaps arising due to regularity in the activity of the robot, e.g. periodicity due to gait.
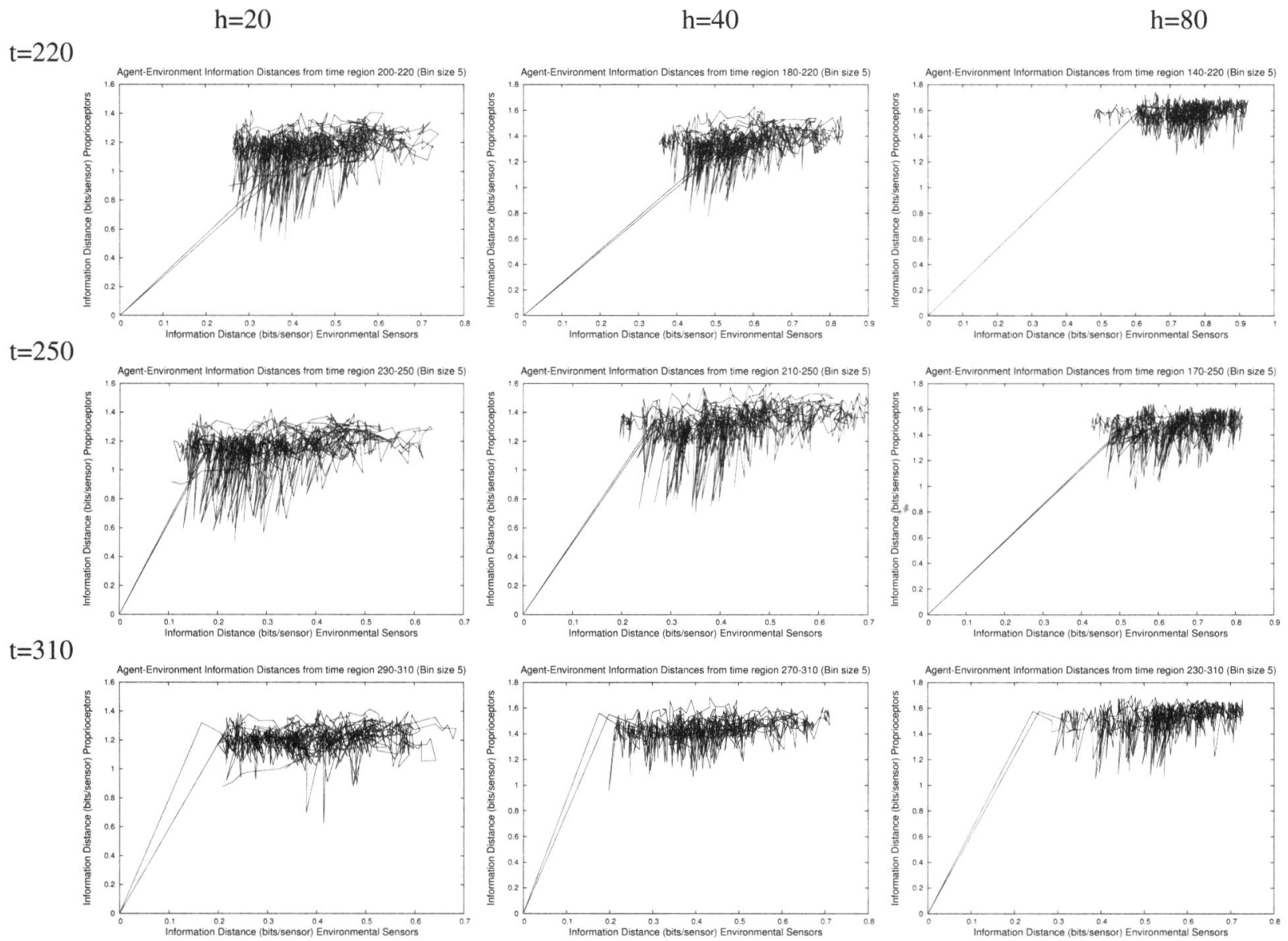

Figure 6: Local picture at experience $E_{h}\left(t_{0}\right)$, the temporal region ending at timestep $t_{0}$ with temporal horizon $h$. The trajectory in experiential space is plotted by giving sensory and motor average experiential distances from $E\left(t_{0}\right)$ for regions ending at timestep $t_{0}=220,250,310$ (top to bottom) with horizons $h$ of 20,40, and 80 timesteps (hundred millisecond time units). See section 2.6. Vertical axis and horizontal axes are in bits per proprioceptor and bits per exteroceptor, respectively, measured by metrics $\bar{D}^{S}$ and $\bar{D}^{M}$. Distances of any experience $E_{h}(t)$ to $E_{h}\left(t_{0}\right)$ are accurately reflected in the local picture as a weighted sum of $x$ - and y-coordinates. Distances between other points are generally not reflected accurately but are guaranteed to be bounded above by the sum of their distances to $E_{h}\left(t_{0}\right)$ as a consequence of the triangle inequality. Robot's behaviour is as described in section 3 and Figure 2. There is no experience was very close in the sampled behaviour to the 9 experiences examined via their local pictures - although nearest experiences usually seemed similar to human observers. 